\title{
3 Research Square

\section{The diagnostic performance of optical coherence tomography and optical coherence tomography angiography for early primary open-angle glaucoma: a cross-sectional study}

\section{Yanjie Li ( $\square$ liyanjie-0311@126.com )}

First Hospital of Shanxi Medical University https://orcid.org/0000-0002-0359-6925

Weishai Liu

First Hospital of Shanxi Medical University

\section{Zichao Bai}

First Hospital of Shanxi Medical University

\section{Rongxia Cao}

First Hospital of Shanxi Medical University

Haihua Ren

First Hospital of Shanxi Medical University

\section{Research article}

Keywords: primary open angle glaucoma, optical coherence tomography, optical coherence tomography angiography, diagnostic utility

Posted Date: October 11th, 2019

DOI: https://doi.org/10.21203/rs.2.15959/v1

License: (c) (i) This work is licensed under a Creative Commons Attribution 4.0 International License.

Read Full License 


\section{Abstract}

Background: Primary open angle glaucoma (POAG) is often delayed in diagnosis and treatment, as it has no acute attack period, and symptoms usually appear at a relatively late stage. Optical coherence tomography angiography (OCTA) on the basis of Carl Zeiss optical coherence tomography with the support of FOURM platform is recently available in China. It is reportedly more sensitive to POAG. We reported the diagnostic utilities of OCTA and OCT which is already in clinical practice.

Methods: 15 patients (23 eyes) with early POAG as observation group and 30 health people (30 eyes) as normal control group were enrolled in this cross-sectional study. OCTA-based superficial macula vessel density, superficial macula perfusion density, superficial optic disc vessel density, superficial optic disc perfusion density and OCT-based macula thickness, ganglion cell complex (GCC) thickness and retinal nerve fiber layer (RNFL) thickness were recorded in the two groups. Independent sample t-test and receiver operating characteristic curve were used for statistical analysis. Area under the receiver operating characteristic curves (AUCs) were used to measure the diagnostic utility.

Results: The optimal diagnostic utility parameters were the superficial vessel density and perfusion density in the macula (except the center of macula), and the AUCs were above 0.97 . Followed by the superficial vessel density and perfusion density in the optic disc area. The OCT-based diagnostic utility parameters were generally lower than that mentioned above, the top three parameters were the inferior RNFL thickness (AUC=0.919), the superior GCC thickness (AUC=0.919) and the inferior GCC thickness $(A \cup C=0.908)$.

Conclusions: Changes of thickness in macula, GCC, RNFL, and changes of superficial vessel density and perfusion density in the optic disc or in macula can be detected by OCT or by OCTA in early POAG. The OCTA-based diagnostic utility was generally higher than the OCT-based diagnostic utility.

\section{Background}

Glaucoma is a group of progressive optic nerve diseases closely related to ganglion cell loss and optic disc change, and accompanying damage to the visual field (VF). It is mainly divided into primary open angle glaucoma (POAG) and primary angle-closure glaucoma (PACG)[1]. POAG, unfortunately, is often delayed in treatment, as it has no acute attack period, and symptoms usually appear at a relatively late stage[2, 3]. Therefore, early diagnosis and treatment of glaucoma as well as close monitoring are crucial for POAG. The etiology and pathogenesis of POAG are not well understood so far. Studies have shown that patients with POAG had a sharply decreased diastolic blood flow of the ophthalmic artery, a significant decreased systolic and diastolic blood flow velocity of the posterior ciliary artery, and an obviously increased resistance index (RI), all of which indicated that the POAG patients had a short supply of blood flow to the posterior ciliary artery, furtherly suggesting insufficient supply to optic disc and choroid. Meanwhile, The systolic and diastolic blood flow velocities of the central retinal artery (CRA) were also significantly decreased, diastolic blood flow of the part of individuals even zero, similarly 
indicating that the central retinal artery of patients with POAG had a low flow velocity and high resistance state, and the blood supply to the surface of the optic disc and to the inner layer of the retina was obviously insufficient[4]. Optical coherence tomography angiography (OCTA) is an imaging technology developed on the basis of spectral domain OCT (SD-OCT), which can detect the retinal microvascular noninvasively in a short time without mydriasis. In addition, it can also provide three-dimensional images, which offer researchers a new method to study the morphology of blood vessels more intuitively and make an accurate qualitative and quantitative analysis of them. All in all, OCTA will provide researchers with a possibility to study and early diagnosis for POAG[5]. In current study, SD-OCT was used to detect macular thickness, RNFL thickness and GCC thickness, and OCTA was used to detect superficial macular vessel density and perfusion density, superficial optic disc vessel density and perfusion density. Calculating the diagnostic efficacy of each parameter (presented as AUROC) was to provide a theoretical basis for the application of SD-OCT and OCTA in the diagnosis and evaluation of early POAG.

\section{Methods}

This was a cross-sectional observational study. At the Department of Ophthalmology of the First Hospital of Shanxi Medical University in Shanxi, republic of China, between October 2018 and June 2019. Written informed consent was obtained from all participants and the study was approved by the Shanxi Medical University's Ethics Committee, and the methodology adheres to the tenets of the Declaration of Helsinki for research involving human subjects.

\section{Participants}

Participants of the study enrolled POAG patients (including 6 males (8 eyes) and 9 females (15 eyes); aged 24-50 (35.00 \pm 10.34$)$ ) and control subjects (including 13 males (13 eyes) and 17 females (17 eyes); aged 22-58 (36.10 \pm 10.85$)$ ). All participants underwent an extensive ophthalmological examination, including slit-lamp biomicroscopy, best-corrected visual acuity, intraocular pressure (IOP), dilated fundus examination, gonioscopy, simultaneous stereophotography of the optic disc, VF testing by standard automated perimetry (SAP, Octopus 101 Perimeter, G2 program, Interzeag, Switzerland), SD-OCT and OCTA imaging (Cirrus HD-OCT 5000, Carl Zeiss Meditec Inc, Germany).

POAG patients inclusion criteria were age $\geq 18$ years, open angles on gonioscopy, glaucomatous changes on optic nerve head examination (neuro-retinal rim narrowing, notching), mean deviation (MD) $>-6 \mathrm{~dB}, \mathrm{a}$ best-corrected visual acuity of $0.3 \mathrm{In}$ logMAR or better, a spherical refraction within \pm 5.0 diopters (D) \and cylinder correction within $\pm 3.0 \mathrm{D}$. POAG patients exclusion criteria were (1) hypertensive or diabetic retinopathy; (2) diagnosis of retinopathy; (3) poor fixation ability (4) SD-OCT or OCTA scans quality $\leq 4$; (5) History of ocular trauma; (6) history of uveitis; (7) history of intraocular surgery; (8) using drugs that may affect the retinal microcirculation; and (9) unreliable VFs (reliable VFs were defined as fixation losses less than $20 \%$ and the false-positive and false-negative response rates less than $15 \%$ ). Healthy eyes had 
(1) IOP $<21 \mathrm{mmHg}$ with no history of elevated IOP; (2) normal appearing optic disc, intact neuroretinal rim;

(3) no family history of glaucoma; and (4) normal anterior segment on clinical examination.

\section{OCT and OCTA examination}

All subjects underwent OCT and OCTA imaging using the Cirrus HD-OCT 5000 Review Software V.10 (Carl Zeiss Meditec Inc.). The optical micro-angiography (OMAG) algorithm was used to capture the dynamic motion of the red blood cells and provide a high-resolution 3D visualization of perfused retinal vasculature. The macular area thickness and the GCC thickness were scanned using the Macular Cube $512 \times 128$ procedure, the RNFL thickness scanning was performed using the Optic Disc Cube $200 \times 200$ procedure, and the optic disc and macular area OCTA scanning were performed using the Angiography $6 \times 6 \mathrm{~mm}$ procedure. The thickness of the RNFL was calculated by the ONH and RNFL OU Analysis mode of the system, the thickness of the GCC was calculated by the Ganglion Cell OU Analysis mode, and the analysis of OCTA (including vessel density and perfusion density analysis on the superficial layer of the optic disc area and the macular area) was calculated by the Angiography Analysis mode on the FOURM platform provided by Carl Zeiss Meditec incorporation. Since the current software version has not offered an analysis of vessel density and perfusion density of deep layer retinal vessels, data of the deep layer retinal vascular density and perfusion density was not available so far. Moreover, FORUM platform only offered the center, inner ring, outer ring and whole area of the vascular density analysis and perfusion density analysis, when the Angiography $6 \times 6 \mathrm{~mm}$ procedure was once chosen. As no more detailed zoning was provided, the vascular density and perfusion density in each sector are not available. All the operation was performed by the same skilled technician.

\section{Statistical Analysis}

Shapiro-Wilk test was used to test for the normality distribution of continuous variables. The demographic data were expressed as mean \pm standard deviation (SD) for continuous variables and frequencies for categorical variables. Mean and $95 \%$ confident interval $(\mathrm{Cl})$ were computed for other normally distributed variables. Continuous variables were analyzed by independent sample T test; Categorical variables were compared using the chi-square test. Area under the receiver operating characteristic curves (AUROC) were used to describe the utility of each parameter to discriminate glaucomatous eyes from the control eyes. An automatic resampling procedure was also used $(n=1000) \square$ as measurements of bilateral eyes nested within subject are more likely to be correlated. Statistical analyses were performed using statistical software (Graphpad Prism7, GraphPad Software, CA, USA). A P-value of $\leq 0.05$ was considered statistically significant.

\section{Results}

A total of 45 subjects (53 eyes), consisting of 15 early POAG subjects (23 eyes), 30 normal health subjects (30 eyes) were included in this study. Demographic and ophthalmic characteristics of the study 
subjects are summarized in Table 1. There was no statistically significant difference in terms of age and gender between the two groups (both $P$ values $>0.05$ ). The IOP was significant higher in the early POAG subjects as compared with the control subjects $(P<0.001)$. The two groups also differed by VFs $(\mathrm{P}<0.001)$.

Table 2 summarizes the measurements of OCT parameters of the two groups. Significantly thicker macula was observed in healthy eyes compared with the early POAG eyes. Parameters of macular area thickness in the inner ring (temporal, nasal, superior, inferior) and the outer ring (nasal, superior, inferior) were found had statistically significant differences (all $P$ values $<0.05$ ). No statistically significant difference, However, was found at the center of macular area thickness $(P=0.990)$ or at the temporal part of outer ring $(P=0.312)$ between the two groups. The GCC thickness in the normal control group was significantly higher than that in the early POAG group in all six sectors (temporal, superotemporal, inferotemporal, nasal, superonasal, inferonasal) (all $P$ values $<0$. 05). Besides, the RNFL thickness in the normal health eyes was significantly higher than that in the early POAG eyes, and there were statistically significant differences both in the superior hemifield and inferior hemifield (both $P$ values $<0.05$ ).

Table 3 summarizes the measurements of OCTA parameters in the macular area for the two groups. The calculated results showed that no statistically significant difference was found in central macula vessel density $(P=0.087)$ and in central macula perfusion density $(P=0.126)$ between the early POAG eyes and healthy eyes. Vascular density (in the inner ring, outer ring, and whole en face) and perfusion density (in the inner ring, outer ring, and whole en face) were all found significantly higher in the normal control subjects than that in the early POAG subjects (all P values<0. 05). (Figure 1 )

Table 4 summarizes the measurements of OCTA parameters in the optic disc area for the two groups. Vascular density (in the center, inner ring, outer ring, and whole en face) and perfusion density (in the center, inner ring, and whole en face) were all found statistically significant differences between the normal control subjects and the early POAG subjects (all $P$ values $<0.05$ ). However, the final results revealed that there was no statistically significant difference in outer ring perfusion density $(P=0.185)$ between the early POAG eyes and healthy eyes.

The AUROCs of all the parameters to differentiate early POAG from healthy control eyes are shown in Table 5. Among these AUROCs, 6 parameters' AUROC ( thickness of the central macular area, thickness of the inferior sector of the inner ring, thickness of the temporal sector of the outer ring, the central macula vessel density, the central macula perfusion density, and the perfusion density of the outer ring of the optic disc area) showed no statistically significant difference (all $P$ values $>0.05$ ). Other parameters' AUROC were found had statistically significant differences to discriminate early POAG from healthy eyes (all $\mathrm{P}<0.05$ ). The parameters with the highest diagnostic utilities were the vascular density in the macular area (except the macular center), and the AUROC reached 0.98 . The diagnostic utilities of perfusion density in the macular area (except the macular center) were comparable to that in the macular area (except the macular center), and the AUROC were above 0.97 . The diagnostic abilities of vascular density in the optic disc area were put at second place, and the optimal parameter was the inner ring vascular 
density, and its AUROC reached 0.97. The diagnostic abilities of perfusion density in the optic disc area were slightly worse than vessel density's in the optic disc area. The optimal parameter was the central optic disc area perfusion density, and its AUC was 0.95. The diagnostic abilities of OCT-based parameters were generally worse than that of OCTA-based parameters, the optimal three parameters were the inferior hemifield RNFL thickness (AUC = 0.919), superior GCC thickness (AUC =0.919), and the inferior GCC thickness $(A \cup C=0.908)$. (Figure 2$)$

\section{Discussion}

POAG is a chronic optic neuropathy characterized by progressive apoptosis of the retinal ganglion cells and elevated intraocular pressure. The two main theories of optic nerve damage mechanism are IOPinduced mechanical injury theory and vascular ischemia theory[3]. Although the IOP-induced mechanical injury theory is generally accepted, in some patients with normal intraocular pressure, the observable excavated narrow-rim ONH appearance and VF defects are also presented. Meanwhile, some glaucoma patients persist in suffering optic disc and VF damages even after using drugs or surgery to control intraocular pressure, suggesting that there are other factors affecting the occurrence of POAG, and the focus on vessel-perfusion changes is one of its research hotspots. There are many technologies to document attenuation in ocular blood flow and dropout of retinal microvascular in glaucoma. However, they have played a limited role in elucidating the mechanism of microvasculature damage. Color doppler ultrasound Imaging (CDI) was once considered the most ideal non-invasive imaging technology, but CDI can only measure blood velocity not the actual blood flow volume. Mansouri et al found that the orbital blood flow in POAG patients was impaired, but the results of these kind of studies were not completely consistent, some even oppositely. The main reason is that CDI relies on the operator, hence, resulting in low repeatability of measurements[6]. OCTA is a new non-invasive, fast and quantitative blood flow detection technology based on OCT, which is mainly used for retinal choroidal blood flow imaging. The emergence of OCTA provides an important research tool for us to figure out the relationship between retinal neuron and axonal loss and blood flow changes, which helps us to understand the pathogenesis of glaucoma from the perspective of vessel density changes.

The thinning of the thickness of the macular area and RNFL, and the loss of GCC usually occur in the early stage of glaucoma. A number of studies have substantiated the fact[7, 8]. However, there are relatively few studies on the vascular density and perfusion density in the macular area and optic disc areas. We have measured the vascular density and perfusion density in the macular area and in the optic disc area, and made a systematical comparison with the widely used macular area thickness, RNFL thickness, and GCC thickness.

In this study, we used the Angiography $6 \times 6 \mathrm{~mm}$ program, which has a wider scan range than Angiography $3 \times 3 \mathrm{~mm}$, which offer more indicators for the diagnosis of early glaucoma. In addition, it has been reported that $6 \times 6 \mathrm{~mm}$ macula scans showed higher diagnostic accuracy compared with $3 \times 3 \mathrm{~mm}$ scans for differentiating between healthy and glaucoma eyes because the most vulnerable macula areas to glaucoma lie mostly outside the central $3 \times 3 \mathrm{~mm}[9,10]$. Compared with Angiography $8 \times 8 \mathrm{~mm}$ program, 
Angiography $6 \times 6 \mathrm{~mm}$ program provides higher resolution scan pictures. All in all, Angiography $6 \times 6 \mathrm{~mm}$ program ensured that we get as many parameters as possible, at the same time, obtain reliable scan quality. In previous studies, reports on the vessel density in the macular area or in the optic disc area often referred as whole image vessel density, and there was no differentiation of the center, the inner ring, and the outer ring. In this study, we found that the vessel density and perfusion density in the central part of the macular area of early glaucoma patients had no statistically significant difference compared to that of normal control eyes. Excluding the center of the macular area from the whole en face scan would be helpful to reduce the error of evaluating the vessel density and perfusion density. To the best of our knowledge, there are relatively few reports focusing on perfusion density to date which is obtained by depicting the width of the blood vessel and calculating the density of blood vessel coverage in the scan region, which can better reflect the perfusion volume of blood flow in the blood vessels. In this study, we also found that the perfusion density in both the macular area and the optic disc area was statistically different between the early POAG group and the normal control group and the diagnostic efficiency was high (except for the outer ring of optic disc area).

Some of the results of this study are consistent with previous findings[11-13]. The thickness of the macular area (except the center of the macular area, superior sector of the inner ring, temporal sector of the outer ring), GCC thickness, and RNFL thickness the vessel density and perfusion density in the optic disc area and the vascular density and perfusion density in the macular area in the early POAG were significantly reduced, compared with the normal control eyes. However, the difference is that this study showed that the diagnostic abilities in the macular area and in optic disc area (including vessel density and perfusion density) were higher than that of Rao, Hou and Zhong[14-16]. We consider the following points to be relevant. First, different imaging devices usually show inconsistent imaging effects, while the imaging quality affects the calculation of vessel density, macular thickness and GCC thickness $[17,18]$. The ZEISS Cirrus HD-OCT 5000 with AngioPlex employed in the study was with the support of the OMAG algorithm, while the AngioVue imaging system applied in most of the published papers uses the SSADA algorithm, which would make a difference in the calculation and comparison of blood vessel density. What's more, different devices and software versions have diverse location for superficial retinal layer vessels, mainly due to the difference in the definition of the position of the inner plexiform layer (IPL). A report by Spaide et al has confirmed that different layered positioning can make a great difference in data[19]. In the current study, we opted for the default AngioPlex automatic layered imaging setup. the external limit of the superficial layer retinal vessels is an approximate position of the IPL, which is estimated by the following formula: $Z_{I P L}=Z_{I L M}+70 \% \times\left(T_{I L M-O P L}\right) \cdot Z_{I P L}$ is the estimated external limit of the IPL; $Z_{\text {ILM }}$ is the boundary position of the internal limiting membrane (ILM); and $T_{\text {ILM-OPL }}$ is the thickness between the ILM and the outer plexiform layer (OPL)[20]. While the most common used AngioVue imaging system defines superficial layer retinal vessels as ILM to IPL-10 $\mu \mathrm{m}$. Second, different classes of topical anti-glaucoma medications could have affected vessel density. Moreover, the age of the normal control group (35.00 \pm 10.34$)$ and the age of the early POAG group $(36.10 \pm 10.85)$ were significantly less than that of the previous reports (the average age was above 60). Both Jian's report and Lin's report revealed that the retinal blood flow index decreases with age in healthy people, especially in 
people over 60 years old. Similarly, vessel density decreases significantly with age in all areas of the retina, which confirmed healthy young people with higher vessel density, compared with the elderly[21, 22]. Therefore, when glaucoma occurs, the decline in vascular density, perfusion density and other parameters of younger POAG patients will be more obvious, due to the higher baseline. This can partly explain why our results showed better diagnostic performance than that of previous reports. Hence, we speculate that OCTA has higher diagnostic value for young patients with early POAG.

There are also some limitations to the current study. Although 30 healthy eyes were included in the healthy control group in order to obtain a more reliable reference, the sample size was still small. Moreover, patients who were over 60 years old were not included in this study. Inclusion of patients in the older age group would be more conducive to explaining the differences in diagnostic performance of macular and optic disc areas in different age groups. In addition, we didn't analyze deep retinal layer blood vessels, for the device has not been able to obtain deep retinal vascular data. After the software update in the future, more available parameters will help us to further understand the pathogenesis of glaucoma. Finally, since this was a cross-sectional study, we are not be able to evaluate the diagnostic value in terms of the progression of POAG. A longitudinal study will be helpful to document the changes of microvascular in progression of glaucoma.

\section{Conclusion}

Changes in macular thickness, GCC thickness, RNFL thickness, superficial vessel density and perfusion density in the optic disc or in macular area can be detected by OCT or OCTA in early POAG. OCTA-based superficial macular vessel density and perfusion density had the highest diagnostic utility. The OCTbased diagnostic utility parameters were generally lower than the OCTA-based diagnostic utility parameters. OCTA has an important clinical application value in diagnosis and evaluation for early POAG.

\section{Abbreviations}

OCTA: Optical coherence tomography angiography

POAG: Primary open angle glaucoma

PACG: primary angle-closure glaucoma

GCC: Ganglion cell complex

$R N F L$ : retinal nerve fiber layer

OMAG: Optical micro-angiography

OPL: Outer plexiform layer 
IPL: Inner plexiform layer

ILM: Inner limit membrane

CDI: Color doppler ultrasound Imaging

IOP: Intraocular pressure

AUROC: Area under the receiver operating characteristic curves

\section{Declarations}

\section{Acknowledgements}

We are grateful to Dr. Guilan Tang for her assistance with patient referral.

\section{Funding}

This work was financially Supported by Key Research and Development (R\&D) Projects of Shanxi Province (201803D31095)

\section{Author information}

\section{Affiliations}

Department of ophthalmology, the first hospital of Shanxi medical university, Taiyuan City, Shanxi Province, China

Yanjie Li, Weishai Liu, Zichao Bai, Rongxia Cao

Shanxi medical university, Taiyuan City, Shanxi Province, China

Haihua Ren

\section{Contributions}

YL and WL designed the study and wrote the manuscript. ZB and RC collected and analyzed the data. HR performed statistical analysis. All authors read and approve the final manuscript.

\section{Corresponding author}

Correspondence to Yanjie $\mathrm{Li}$ 


\section{Ethics declarations}

\section{Ethics approval and consent to participate}

This study was approved by the Ethics Committee of the First hospital of Shanxi Medical university. A written informed consent was obtained from each subject.

\section{Consent for publication}

Written informed consent for publication was obtained from 1 subject whose OCTA images were present in Fig. 1.

\section{Competing interests}

The authors declare that they have no competing interests

\section{References}

1.Quigley HA, Broman AT: The number of people with glaucoma worldwide in 2010 and 2020. The British journal of ophthalmology 2006, 90(3):262-267.

2.Leite MT, Sakata LM, Medeiros FA: Managing glaucoma in developing countries. Arquivos Brasileiros De Oftalmologia 2011, 74(2):83-84.

3.Weinreb R, Khaw P: Primary open-angle glaucoma. New England Journal of Medicine 2004, 363(9422):1711.

4.Akarsu C, Bilgili MY: Color Doppler imaging in ocular hypertension and open-angle glaucoma. Graefes Archive for Clinical Experimental Ophthalmology 2004, 242(2):125-129.

5.Werner AC, Shen LQ: A Review of OCT Angiography in Glaucoma. Seminars in ophthalmology 2019, 34(4):279-286.

6.Tokayer J, Jia Y, Dhalla AH, Huang D: Blood flow velocity quantification using split-spectrum amplitudedecorrelation angiography with optical coherence tomography. Biomedical optics express 2013, 4(10):1909-1924.

7.Penteado RC, Zangwill LM, Daga FB, Saunders LJ, Weinreb RN: Optical Coherence Tomography Angiography Macular Vascular Density Measurements and the Central 10-2 Visual Field in Glaucoma. Journal of Glaucoma 2018, 27(6):1. 
8.Alluwimi MS, Swanson WH, King BJ: Identifying Glaucomatous Damage to the Macula. Optometry and vision science: official publication of the American Academy of Optometry 2018, 95(2):96-105.

9.Hood DC, Raza AS, de Moraes CG, Liebmann JM, Ritch R: Glaucomatous damage of the macula. Progress in retinal and eye research 2013, 32:1-21.

10.Hood DC: Improving our understanding, and detection, of glaucomatous damage: An approach based upon optical coherence tomography (OCT). Progress in retinal and eye research 2017, 57:46-75.

11.Park SB, Sung KR, Kang SY, Kim KR, Kook MS: Comparison of glaucoma diagnostic capabilities of Cirrus HD and Stratus optical coherence tomography. Archives of Ophthalmology 2009, 127(12):16031609.

12.Xu H, Yu J, Kong X, Sun X, Jiang C: Macular microvasculature alterations in patients with primary open-angle glaucoma: A cross-sectional study. Medicine 2016, 95(33):e4341.

13. Nakano N, Hangai M, Noma H, Nukada M, Mori S, Morooka S, Takayama K, Kimura Y, Ikeda HO, Akagi T: Macular Imaging in Highly Myopic Eyes With and Without Glaucoma. American Journal of Ophthalmology 2013, 156(3):511-523.

14.Rao HL, Pradhan ZS, Weinreb RN, Reddy HB, Riyazuddin M, Dasari S, Palakurthy M, Puttaiah NK, Rao DAS, Webers CA: Regional comparisons of optical coherence tomography angiography vessel density in primary open angle glaucoma. American Journal of Ophthalmology 2016, 171:75-83.

15.Hou H, Moghimi S, Zangwill LM, Shoji T, Ghahari E, Penteado RC, Akagi T, Manalastas PIC, Weinreb RN: Macula Vessel Density and Thickness in Early Primary Open-Angle Glaucoma. American Journal of Ophthalmology 2019, 199:120-132.

16.Zhong Y, Che H: Detection Value of Optical Coherence Tomography Angiography in Patients with Primary Glaucoma. Recent Advances in Ophthalmology 2018, 38(4):352-356.

17.Iafe NA, Phasukkijwatana N, Chen X, Sarraf D: Retinal Capillary Density and Foveal Avascular Zone Area Are Age-Dependent: Quantitative Analysis Using Optical Coherence Tomography Angiography. Investigative ophthalmology \& visual science 2016, 57(13):5780-5787.

18.von Hanno T, Lade AC, Mathiesen EB, Peto T, Njolstad I, Bertelsen G: Macular thickness in healthy eyes of adults $(N=4508)$ and relation to sex, age and refraction: the Tromso Eye Study (2007-2008). Acta ophthalmologica 2017, 95(3):262-269.

19.Spaide RF, Curcio CA: Evaluation of Segmentation of the Superficial and Deep Vascular Layers of the Retina by Optical Coherence Tomography Angiography Instruments in Normal Eyes. JAMA ophthalmology 2017, 135(3):259-262. 
20.Wei W: Atlas of Optical coherence Tomography Angiography, 1 edn: People's Medical Publishing House; 2016.

21.Lin Y, Jiang H, Liu Y, Rosa Gameiro G, Gregori G, Dong C, Rundek T, Wang J: Age-Related Alterations in Retinal Tissue Perfusion and Volumetric Vessel Density. Investigative ophthalmology \& visual science 2019, 60(2):685-693.

22.Yu J, Jiang C, Wang X, Zhu L, Gu R, Xu H, Jia Y, Huang D, Sun X: Macular perfusion in healthy Chinese: an optical coherence tomography angiogram study. Investigative ophthalmology \& visual science 2015, 56(5):3212-3217.

\section{Tables}

Table 1. Demographics and Ocular Characteristics of Study Population

\begin{tabular}{|c|c|c|c|c|c|}
\hline & $\mathrm{s}(\mathrm{n})$ & & $\mathrm{Sex} \square \mathrm{M} / \mathrm{F} \square$ & $\mathrm{IOP} \square \mathrm{mmHg} \square$ & $\mathrm{MD}$ \\
\hline $\begin{array}{l}\text { Control } \\
\text { POAG } \\
P\end{array}$ & $\begin{array}{l}30 \\
23 \\
\text { NA }\end{array}$ & $\begin{array}{l}36.10 \pm 10.85 \\
35.00 \pm 10.34 \\
0.96\end{array}$ & $\begin{array}{l}13 / 17 \\
6 / 9 \\
0.83\end{array}$ & $\begin{array}{l}14.23 \pm 3.45 \\
20.86 \pm 5.02 \\
<0.001\end{array}$ & $\begin{array}{l}0.04 \pm 0.3 \\
-4.5 \pm 1.2 \\
<0.001\end{array}$ \\
\hline
\end{tabular}

Abbreviations: IOP, intraocular pressure; MD, mean deviation; POAG, primary open-angle glaucoma; M/F, male/female; NA, not available

Table 2. OCT Based Parameters in POAG and Control Eyes $\square$ m): Independent sample T Test

\begin{tabular}{|c|c|c|c|c|}
\hline & Control & POAG & $\mathrm{t}$ & $P$ \\
\hline \multicolumn{5}{|l|}{ Macula thickness } \\
\hline Central & $249.33 \pm 7.57$ & $249.08 \pm 21.24$ & 0.008 & 0.990 \\
\hline Nasal (IR) & $.50 \pm 15.23$ & $310.08 \pm 25.87$ & 2.305 & 0.028 \\
\hline Temporal (IR) & $.14 \pm 10.61$ & $297.88 \pm 21.55$ & 2.266 & 0.031 \\
\hline Superior (IR) & \pm 14.21 & $4 \pm 26.40$ & 2.1 & 0.044 \\
\hline Inferior (IR) & \pm 8.06 & $1 \pm 20.15$ & & 002 \\
\hline Nasal (OR) & \pm 13.80 & $7 \pm 25.41$ & 2.150 & 0.040 \\
\hline ral (OR) & & & & 312 \\
\hline or (OR) & \pm 9.06 & 17.11 & 2.175 & 0.038 \\
\hline Inferior (OR) & & & & 0.012 \\
\hline $\begin{array}{l}\text { GuC lnickness } \\
\text { superior }\end{array}$ & $86.43+3.72$ & $6938+14$ & & 0.004 \\
\hline $\begin{array}{l}\text { infer } \\
\text { infor }\end{array}$ & & & & \\
\hline temporal & & & & 0.017 \\
\hline temporal & & & 3.205 & 0.003 \\
\hline & & & & \\
\hline inferonasal & & & 3.09 & 0.00 \\
\hline \multicolumn{5}{|l|}{ RNFL thickness } \\
\hline superior & $111.33 \pm 11$ & $82.49 \pm 2$ & 3.279 & 0.002 \\
\hline inferior & & $77.83 \pm 28.98$ & 4.904 & -000 \\
\hline
\end{tabular}


Abbreviation: IR, inner ring; OR, outer ring; GCC, ganglion cell complex; RNFL, retinal nerve fiber layer

Table 3. OCTA Based Parameters in Macular area: Independent sample T Test

\begin{tabular}{lllll}
\hline & Control & POAG & t & $P$ \\
\hline Central VD & $8.463 \pm 1.4422$ & $5.010 \pm 5.1624$ & 1.825 & 0.087 \\
Inner ring VD & $17.488 \pm 1.5851$ & $8.960 \pm 4.5625$ & 5.597 & $<0.001$ \\
Outer ring VD & $17.988 \pm 1.9775$ & $10.750 \pm 3.2945$ & 5.446 & $<0.001$ \\
Whole en face VD & $17.625 \pm 1.7417$ & $10.130 \pm 3.4137$ & 5.628 & $<0.001$ \\
Central PD & $0.179 \pm 0.0392$ & $0.108 \pm 0.1193$ & 1.619 & 0.126 \\
Inner ring PD & $0.416 \pm 0.0322$ & $0.214 \pm 0.1197$ & 4.617 & $<0.001$ \\
Outer ring PD & $0.445 \pm 0.0394$ & $0.264 \pm 0.0888$ & 5.346 & $<0.001$ \\
Whole en face PD & $0.430 \pm 0.0374$ & $0.246 \pm 0.0907$ & 5.354 & $<0.001$ \\
\hline
\end{tabular}

Abbreviations: VD, vessel density; PD, perfusion density; POAG, primary open-angle glaucoma

Table 4. OCT Based Parameters in Optic Disc Area: Independent sample T Test

\begin{tabular}{lllll}
\hline & Control & POAG & t & $P$ \\
\hline Central VD & $10.029 \pm 6.1587$ & $0.370 \pm 0.3129$ & 5.022 & $<0.001$ \\
Inner ring VD & $18.257 \pm 1.7444$ & $11.470 \pm 2.9702$ & 5.398 & $<0.001$ \\
Outer ring VD & $18.114 \pm 1.5137$ & $14.520 \pm 3.4730$ & 2.398 & 0.022 \\
Whole en face VD & $17.929 \pm 1.1280$ & $13.430 \pm 3.2187$ & 3.017 & 0.003 \\
Central PD & $0.211 \pm 0.1800$ & $0.010 \pm 0.0064$ & 2.958 & 0.004 \\
Inner ring PD & $0.443 \pm 0.0606$ & $0.317 \pm 0.0690$ & 4.126 & $<0.001$ \\
Outer ring PD & $0.429 \pm 0.0576$ & $0.387 \pm 0.0699$ & 1.306 & 0.181 \\
Whole en face PD & $0.435 \pm 0.0390$ & $0.360 \pm 0.0661$ & 2.674 & 0.010 \\
\hline
\end{tabular}

Abbreviations: VD: vessel density; PD: perfusion density

Table 5. Diagnostic Ability of Each Parameter in Differentiating POAG from Control eyes 


\begin{tabular}{lllll}
\hline & AUROC & SE & $95 \%$ CI & $P$ \\
\hline Central & 0.5387 & 0.1055 & $0.3319-0.7455$ & 0.7588 \\
Nasal (IR) & 0.7682 & 0.0845 & $0.6025-0.9339$ & 0.0250 \\
Temporal (IR) & 0.7976 & 0.0796 & $0.6415-0.9537$ & 0.0182 \\
Superior (IR) & 0.7292 & 0.0957 & $0.5416-0.9168$ & 0.0689 \\
Inferior (IR) & 0.8861 & 0.0659 & $0.7370-0.9952$ & 0.0037 \\
Nasal (OR) & 0.7946 & 0.0818 & $0.6344-0.9549$ & 0.0194 \\
Temporal (OR) & 0.6875 & 0.0955 & $0.5003-0.8747$ & 0.1367 \\
Superior (OR) & 0.7589 & 0.0835 & $0.5953-0.9226$ & 0.0399 \\
Inferior (OR) & 0.8274 & 0.0746 & $0.6812-0.9736$ & 0.0094 \\
Superior GCC & 0.9196 & 0.0484 & $0.8248-1.0150$ & 0.0009 \\
Inferior GCC & 0.9077 & 0.0547 & $0.8005-1.0150$ & 0.0012 \\
Superonasal GCC & 0.8333 & 0.0761 & $0.6842-0.9824$ & 0.0082 \\
Inferonasal GCC & 0.8333 & 0.0723 & $0.6917-0.9750$ & 0.0082 \\
superotemporal & 0.8423 & 0.0711 & $0.7030-0.9815$ & 0.0066 \\
inferotemporal & 0.8899 & 0.0592 & $0.7739-1.0060$ & 0.0020 \\
Superior RNFL & 0.8143 & 0.0634 & $0.6901-0.9385$ & 0.0040 \\
Inferior RNFL & 0.9190 & 0.0423 & $0.8362-1.0020$ & 0.0001 \\
Center VD & 0.7125 & 0.1291 & $0.4594-0.9656$ & 0.1309 \\
Inner ring VD & 0.9875 & 0.0200 & $0.9483-1.0270$ & 0.0005 \\
Outer ring VD & 0.9813 & 0.0259 & $0.9305-1.0320$ & 0.0006 \\
Whole en face VD & 0.9875 & 0.0200 & $0.9483-1.0270$ & 0.0005 \\
Center PD & 0.7625 & 0.1179 & $0.5314-0.9936$ & 0.0621 \\
Inner ring PD & 0.9750 & 0.0318 & $0.9128-1.0370$ & 0.0007 \\
Outer ring PD & 0.9875 & 0.0200 & $0.9483-1.0270$ & 0.0005 \\
Whole en face PD & 0.9875 & 0.0200 & $0.9483-1.0270$ & 0.0005 \\
Center VD & 0.9429 & 0.0609 & $0.8235-1.0620$ & 0.0025 \\
Inner ring VD & 0.9714 & 0.0361 & $0.9006-1.0420$ & 0.0013 \\
Outer ring VD & 0.8429 & 0.0956 & $0.6555-1.0300$ & 0.0192 \\
Whole en face VD & 0.9286 & 0.0607 & $0.8095-1.0480$ & 0.0034 \\
Center PD & 0.9506 & 0.0530 & $0.8468-1.0540$ & 0.0013 \\
Inner ring PD & 0.9012 & 0.0717 & $0.7608-1.0420$ & 0.0041 \\
Outer ring PD & 0.6852 & 0.1316 & $0.4273-0.9431$ & 0.1853 \\
Whole en face PD & 0.8395 & 0.0965 & $0.6504-1.029$ & 0.0152 \\
\hline
\end{tabular}

Abbreviations $₫ A U R O C \square$ area under the receiver operating characteristic curve $\triangle \mathrm{SE} \llbracket$ standard error $\square \mathrm{CI} \square$ confidence interval; VD: vessel density; PD: perfusion density; OR: outer ring; IR: inner ring

\section{Figures}
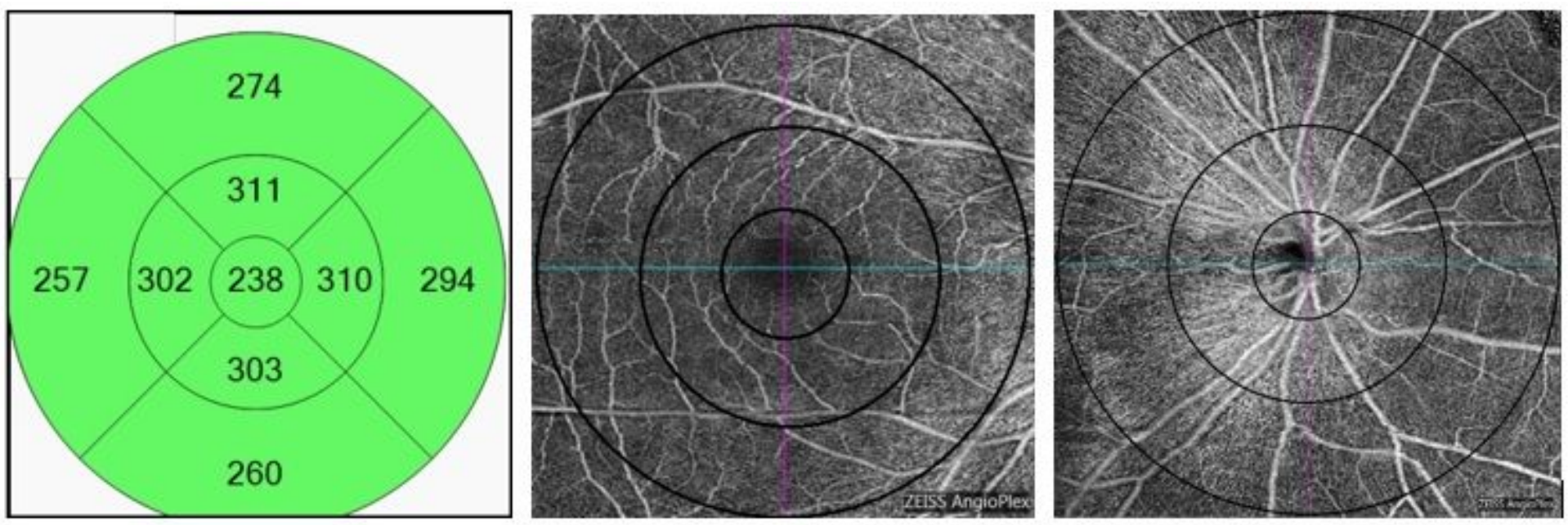
Figure 1

Figure showing the macular area thickness in each sector on a normal eye (Left), optical coherence tomography angiography images of macular area (Middle) and optic disc area (Right).

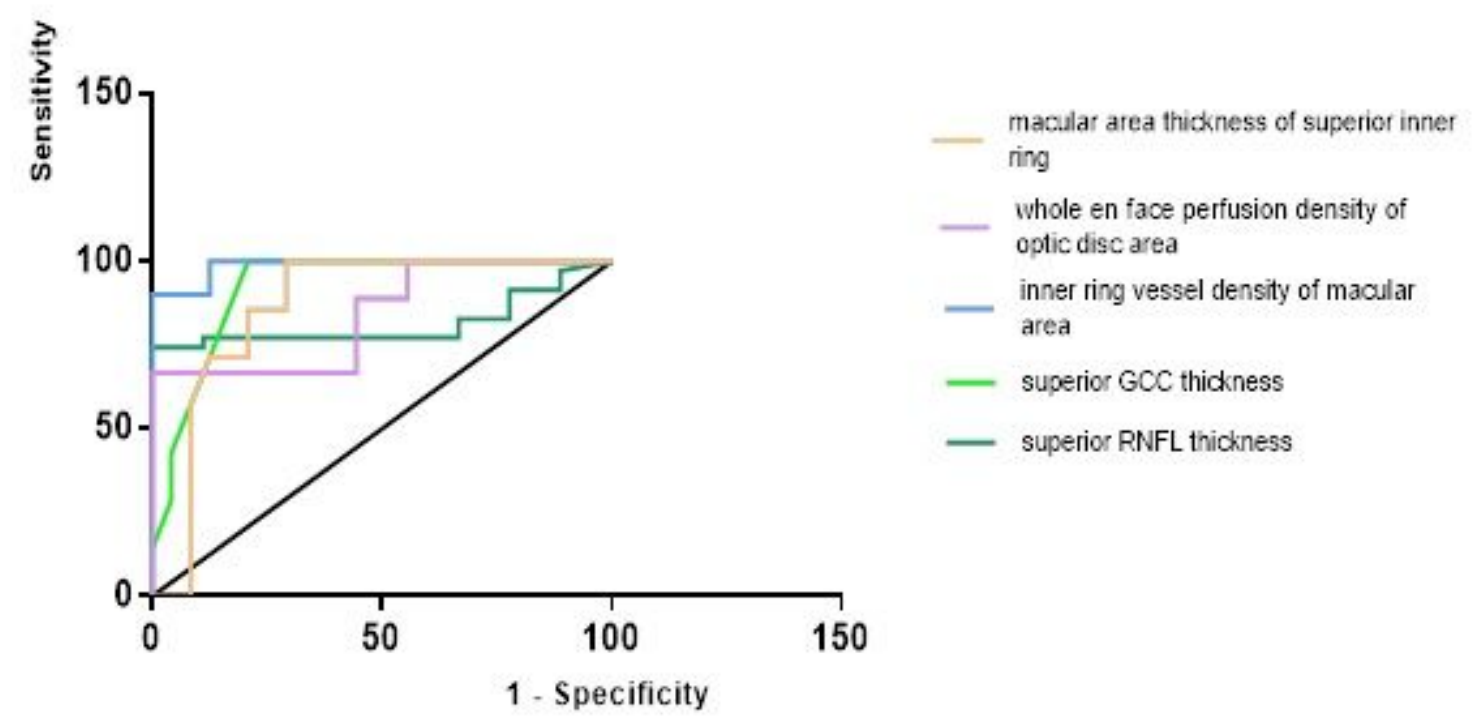

\section{Figure 2}

Receiver operating characteristic curves of superior inner ring macula thickness, whole en face perfusion density of optic disc area, macular inner ring vessel density, superior GCC thickness and superior RNFL thickness 